
\title{
28 Research Square \\ Diffuse peritonitis caused by spontaneous perforation of pyometra: A case report and literature review
}

Hao Xu

Lanzhou University First Affiliated Hospital

\section{Yifan Zhuang}

Zhongshan Hospital Xiamen University

\section{Caiming Wang}

Second People's Hospital of Baiyin city

\section{Yongjie Zhou}

Lanzhou University First Affiliated Hospital

\section{Haofei Chen}

Lanzhou University First Affiliated Hospital

\section{Xin Li}

Lanzhou University First Affiliated Hospital

Wence Zhou ( $\nabla$ zhouwc129@163.com )

Lanzhou University First Affiliated Hospital

\section{Case report}

Keywords: cervical squamous cell carcinoma, diffuse peritonitis, spontaneous perforation, pyometra

Posted Date: January 23rd, 2021

DOl: https://doi.org/10.21203/rs.3.rs-151635/v1

License: (c) (i) This work is licensed under a Creative Commons Attribution 4.0 International License. Read Full License 


\section{Abstract}

\section{Background}

Uterine perforation is commonly caused by iatrogenic injuries, tumours, and traumas. Uterine perforation resulting from infection is less common, and such perforation rarely causes abdominal empyema and diffuse peritonitis. Infectious complications such as shock and multiple organ dysfunction are extremely dangerous and even life threatening. Correct early diagnosis and appropriate treatment can reduce the mortality of pyometra.

\section{Case presentation}

A 62-year-old woman presented to our hospital with persistent abdominal pain for 5 hours. The patient had undergone hysteroscopy six months ago, and a cystic space-occupying lesion of the uterus was found one month ago. Perfect the various examinations, consider the patient as acute diffuse peritonitis Perforation of the digestive tract? Uterine cavity infection. In the emergency department, laparoscopic exploration was performed. During the operation, it was diagnosed as pyometra and perforation of the right uterine fundus. Then, combined with a gynecologist, a hysterectomy with double appendages was performed. The operation went smoothly. The patient had paralytic intestinal obstruction after the operation. After active symptomatic treatment The patient recovered well and was discharged.

Postoperative pathology results were as follows: 1. Moderately differentiated squamous cell carcinoma of the cervix (non-keratinizing type); 2 . Metastatic squamous cell carcinoma of the right ovary; 3. Perforation of the fundus of the uterus, pyometra, and extensive purulent inflammation of the muscle wall.

\section{Conclusions}

Uterine perforation is a rare cause of acute abdomen. Clinicians often don't know enough about this, and they are prone to misdiagnosis and missed diagnosis. For elderly women with abdominal pain and peritonitis, the possibility of empyema perforation should be considered during diagnosis and treatment, and the examination should be improved to avoid misdiagnosis, because correct early diagnosis and appropriate treatment can reduce the mortality of empyema.

\section{Background}

Genital infection in elderly women is a common gynaecological disease, but the resulting empyema is less common; uterine perforation caused by empyema is even rarer, and its complications often conceal the symptoms of the original disease and lead to misdiagnosis [1]. Here, we report a patient who presented with abdominal pain and diffuse peritonitis due to empyema and perforation.

\section{Case Presentation}


The patient, 62 years old, G3P3, with 15 years of menopause, went to the emergency department of our hospital due to persistent abdominal pain for 5 hours. She had undergone hysteroscopy at the local hospital half a year prior. A cystic space-occupying lesion of the uterus was found before January of the present year, and a general surgery consultation and physical examination were requested. The results were as follows: T $36.5^{\circ} \mathrm{C}, \mathrm{P}$ 93/min, R 24 times/min, BP 100/60 mmHg, clear consciousness, poor mood, passive posture, whole abdominal tenderness, rebound pain, and muscle tension. Abdominal puncture was performed to draw out purulent fluid. Routine blood test results were as follows: CRP

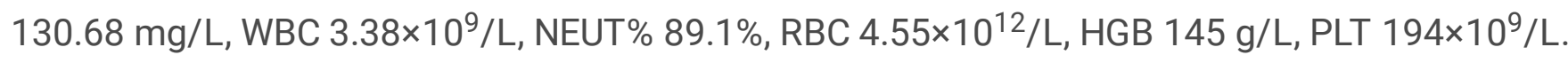
Abdominal standing radiography revealed free gas under the diaphragm and gastrointestinal perforation (Fig. 1a). Ultrasound revealed uterine effusion, pelvic effusion, and ascites (Fig. 1b). Outpatient abdominal CT showed free gas in the abdominal cavity, fluid accumulation in the abdominal cavity and pelvis, possible perforation of the hollow organs, fluid accumulation in the uterine cavity and gas accumulation (Fig. 1c and d). The admission diagnosis was suspected acute diffuse peritonitis with perforation of the digestive tract and uterine cavity infection. A more detailed preoperative examination was conducted, and exploratory laparotomy under laparoscopy was performed. During the operation, 800 $\mathrm{ml}$ of empyema in the abdomen and pus coating the surface of the intestine were observed. There was no perforated lesion in the gastrointestinal tract, and the appendix was not purulent or perforated. Continuing to explore the pelvic cavity, it was found that the uterus was soft, large, and red in colour. There was a rupture of approximately $5 \mathrm{~mm} \times 5 \mathrm{~mm}$ in the left fundus of the uterus. There was a continuous outflow of yellow liquid, which was connected to the abdominal cavity (Fig. 2a). A gynaecologist was requested for surgery, and a uterus plus double attachment resection was performed (Fig. 2b). The abdominal cavity was repeatedly flushed, and 2 abdominal drainage tubes were placed. After the operation, the patient developed paralytic intestinal obstruction. After active symptomatic treatment, the patient recovered well and was discharged. Postoperative pathology results were as follows: 1 . Moderately differentiated squamous cell carcinoma of the cervix (non-keratinizing type); 2 . Metastatic squamous cell carcinoma of the right ovary; 3 . Perforation of the fundus of the uterus, pyometra, and extensive purulent inflammation of the muscle wall (Fig. 2c).

\section{Discussion}

Inflammation of the female reproductive system is common; however, spontaneous uterine perforation due to purulent metritis and pyometra is clinically rare and thus easily overlooked or misdiagnosed. The older the age, the higher is the incidence; poor personal hygiene is the most common causative factor. Postmenopausal women have low oestrogen levels, along with reduced vaginal mucosal keratosis and decreased intracellular glycogen. Inhibiting the production of lactic acid weakens the self-purification effect of the vagina, decreases resistance to infection and causes infection. In addition, the cervix is atrophied, the cervical canal becomes narrow, adhesions easily occur, and the myometrium is atrophied and thinned. Therefore, inflammatory secretions cannot be drained in a timely manner or are not drained smoothly, causing intrauterine empyema, which increases pressure in the uterine cavity, erodes the thinned myometrium, and leads to tissue necrosis and perforation [2, 3]. 
When a patient sees a doctor due to fever, lower abdomen swelling, pain, and increased vaginal discharge, it is easier to reach a diagnosis by gynaecological examination and imaging examination. However, not all patients with empyema have typical symptoms. When treating digestive system symptoms, it is easily misdiagnosed as a perforation of the digestive tract in clinical practice, especially in elderly patients [4-8].

Elderly individuals are slow to respond and exhibit memory decline, and most of them cannot clearly describe their medical history. In addition, they exhibit low touch and pain thresholds and a poor response to inflammation. The clinical symptoms are more concealed, and the signs are not typical. Abdominal wall relaxation, abdominal muscle atrophy, fat thickening, and abdominal muscle tension are not obvious, and the symptoms and signs are less severe than the actual lesions. Moreover, elderly women rarely undergo gynaecological examinations, and the older they are, the fewer physical examinations they undergo. As a result, most elderly women cannot seek timely medical treatment when they are sick, consequently delaying diagnosis and treatment. The difference between uterine and gastrointestinal perforations can be distinguished by the following points: $\otimes$ Carefully solicit the medical history. Uterine abscess perforation is more common in elderly menopausal women; the early symptoms are only increased leucorrhoea with lower abdominal pain, which is tolerable and thus not paid attention to, which delays recognition of the condition. However, most upper gastrointestinal perforations have a history of ulcers, most of which involve acute abdominal pain after eating or a sudden onset of abdominal pain; the starting point is mostly in the upper abdomen, from which the pain spreads rapidly to the whole abdomen, and the upper abdomen is more severe. $\nabla$ Perform abdominal puncture. When the upper gastrointestinal tract is perforated, the extracted fluid will be a grass-green or yellow thin liquid that is odourless and mixed with food residues; microscopic examination reveals a small amount of pus. In contrast, the liquid extracted from a uterine abscess perforation is yellowish white, viscous, and malodourous; microscopic examination reveals pus throughout the full field of view. $\nabla$ If elderly female patients present a large amount of fluid in the abdominal cavity and free gas in the septum within a short period of time, perforation of the uterine abscess should be considered. In contrast, if perforation of the upper gastrointestinal tract has occurred, a large amount of fluid in the abdominal cavity is unlikely to accumulate within a short period of time.

At the same time, postmenopausal uterine inflammation should be differentiated from endometrial cancer, fallopian tube cancer, and cervical cancer. Because endometrial cancer is a tumour that is more common in elderly women, it often manifests as various types of uterine bleeding, abnormal vaginal discharge and pain. The lesions may be formed due to poor drainage in the lower uterus or invasion of the cervical canal. Haemorrhage or empyema may be present in the uterine cavity. The postoperative examination of the present patient showed a moderately differentiated squamous cell carcinoma of the cervix, indicating that when cervical tumours cause poor drainage of the cervical canal, they can also cause empyema and even perforation into the uterine cavity.

Elderly patients with pyometra and spontaneous uterine perforation show a rapid onset, rapid changes, and severe illness. If they are not treated in time, septic shock and multiple organ dysfunction may occur, 
and the original disease may even be life-threatening. According to the literature, the median age of rupture and perforation of empyema is 73.8 years; for elderly women with complicated diseases, the mortality rate is $25-40 \%[1,6,7,9]$.

The principle of treatment of this disease is to carry out blood, body fluid or faecal bacterial culture and drug sensitivity tests on the basis of symptomatic and supportive therapy. Adequate and sensitive antibiotics and antifungal drugs should be provided. When intra-abdominal infection is suspected and the formation of an abscess is confirmed by examination, surgical exploration and drainage should be performed in a timely manner. Advanced age should not be considered a contraindication to surgery; however, elderly patients often have other diseases. In this population, surgery and anaesthesia are extremely risky, the perioperative condition changes drastically, and there is even a risk of death. Therefore, patients' families should be informed in detail regarding possible complications during the perioperative period and be encouraged to consider surgical treatment. The surgery should have a short duration, incur minimal trauma, and involve simple and safe procedures.

\section{Conclusion}

Uterine perforation is a rare cause of acute abdomen. Clinicians often do not know enough about this condition, and consequently, they are prone to misdiagnosis and missed diagnosis. For elderly women with abdominal pain and peritonitis, the possibility of empyema perforation should be considered during diagnosis and treatment, and the examination should be improved to avoid misdiagnosis because correct early diagnosis and appropriate treatment can reduce the mortality of empyema. At the same time, strengthening health education for elderly women; performing regular gynaecological examinations in this population; and focusing on prevention, early treatment, and early treatment can avoid the serious consequences of uterine perforation caused by pyometra.

\section{Abbreviations}

BP: Blood pressure; CRP:C-reaction protein; WBC:White blood cell; NEUT:Neutrophil; RBC:Red blood cell; HGB:Hemoglobin; PLT:Platelets; CT:Computed tomography

\section{Declarations}

\section{Ethics approval and consent to participate}

Since this is a case report, approval from the local ethical committee is not necessary.

\section{Consent for publication}

The patient signed consent for publication of data (including individual details and images).

\section{Availability of data and materials}


All data generated or analyzed during this study are included in this published article.

\section{Competing interests}

The authors declare that they have no competing interests.

\section{Funding}

This work was supported, in part, by the Gansu Provincial Science and Technology Key Research and Development Program (17YF1FA128), Gansu Health Industry Research Project (GSWSKY-2015-51), and Fund of the First Hospital of Lanzhou University (Idyyyn2015-10).

\section{Authors' contributions}

WCZ and HX analyzed and interpreted the patient data. HX, WCZ, and YFZ were the major contributors in the writing of the manuscript. CMW, YJZ, HFC, and XL participated in the patient management. All authors read and approved the final manuscript.

\section{Acknowledgements}

Not applicable.

\section{Authors' information}

${ }^{1}$ The Second Department of General Surgery, the First Hospital of Lanzhou University, Lanzhou, Gansu Province, China

${ }^{2}$ The First Clinical Medical School of Lanzhou University, Lanzhou, Gansu Province, China

${ }^{3}$ Department of Gastrointestinal Surgery, Zhongshan Hospital of Xiamen University, Xiamen, Fujian Province, China

${ }^{4}$ Department of Tumour and Anorectal, Second People's Hospital of Baiyin City, Baiyin, Gansu Province, China

\section{References}

1. Balas Ş, Yılmaz KB, Yıldııım SA, Açıkgöz B, Tatar I G, Bayar B, et al. Spontaneous perforation of pyometra: a rare cause of acute abdomen and sepsis. Turk J Surg. 2018;34:342-5.

2. Zhu MJ, Li P. Clinical analysis of misdiagnosis of spontaneous uterus perforation in old women. J Rare Uncommon Dis. 2007;14:31-2.

3. Desai AY, Palande B, Dhabolkar S, Pai VD. Perforative peritonitis-gastrointestinal tract may not always be the source. Indian J Surg. 2017;79:160-2. 
4. Vyas S, Kumar A, Prakash M, Kapoor R, Kumar P, Khandelwal N. Spontaneous perforation of pyometra in a cervical cancer patient: a case report and literature review. Cancer Imaging. 2009;9:124.

5. Yin WB, Wei YH, Liu GW, Zhao XT, Zhang MS, Hu JL, et al. Spontaneous perforation of pyometra presenting as acute abdomen: a rare condition with considerable mortality. Am J Emerg Med. 2016;34:761.e3-5.

6. Kitai T, Okuno K, Ugaki H, Komoto Y, Fujimi S, Takemura M. Spontaneous uterine perforation of pyometra presenting as acute abdomen. Case Rep Obstet Gynecol. 2014;2014:738568.

7. Ikeda M, Takahashi T, Kurachi H. Spontaneous perforation of pyometra: a report of seven cases and review of the literature. Gynecol Obstet Invest. 2013;75:243-9.

8. Ou YC, Lan KC, Lin H, Tsai CC, ChangChien CC. Clinical characteristics of perforated pyometra and impending perforation: specific issues in gynecological emergency. J Obstet Gynaecol Res. 2010;36:661-6.

9. Uno K, Tano S, Yoshihara M, Mayama M, Ukai M, Kishigami Y, et al. A case report and literature review of spontaneous perforation of pyometra. J Emerg Med. 2016;50:e231-6.

\section{Figures}


$\mathbf{a}$

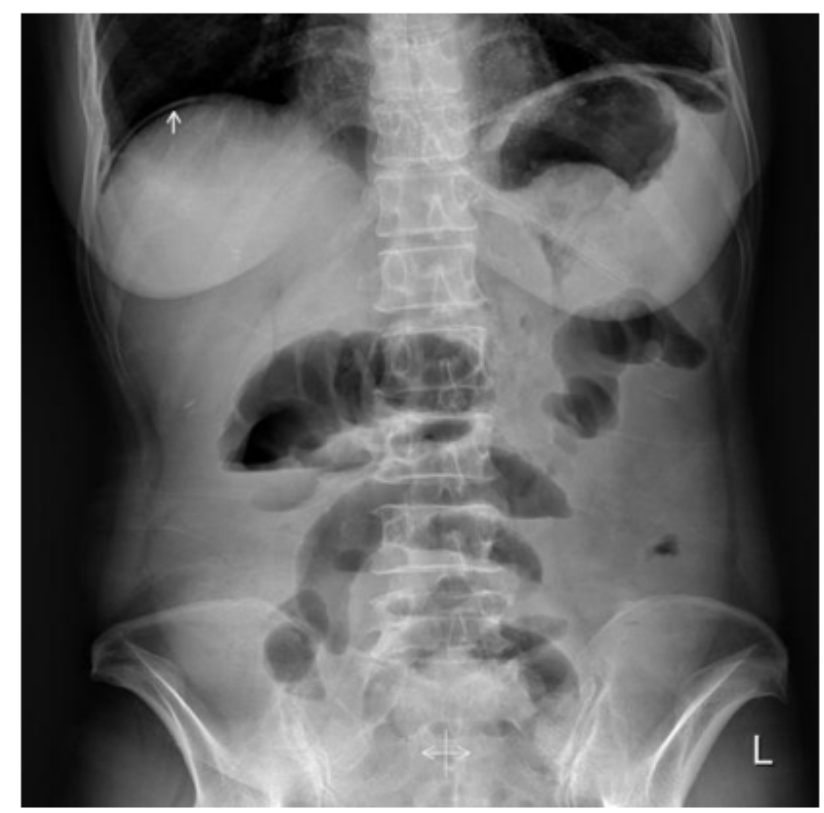

c

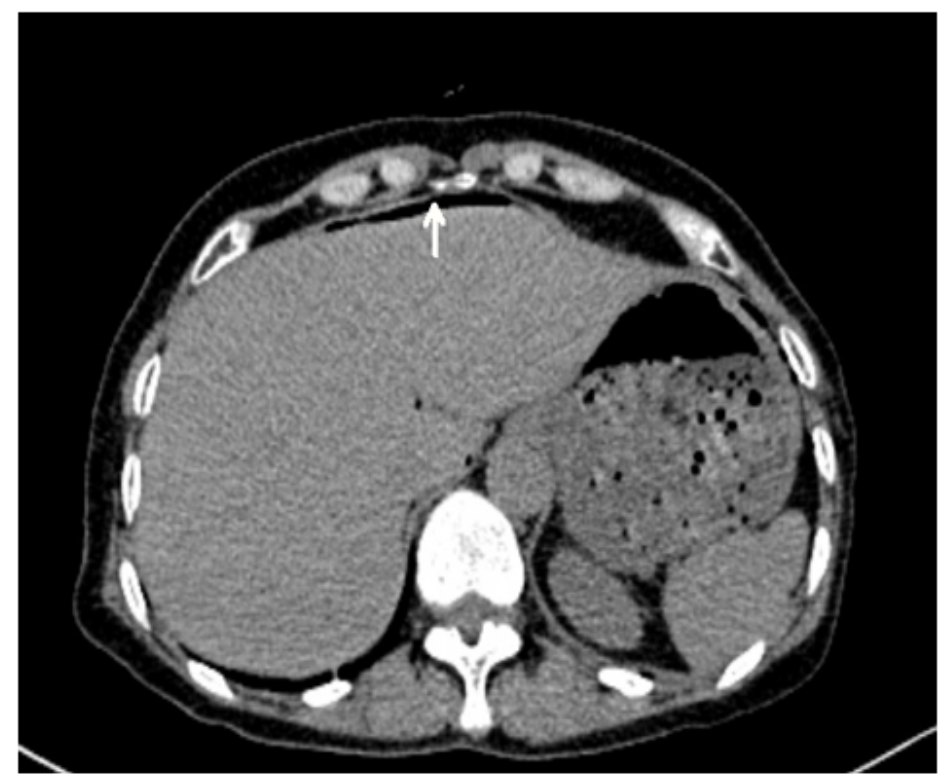

b

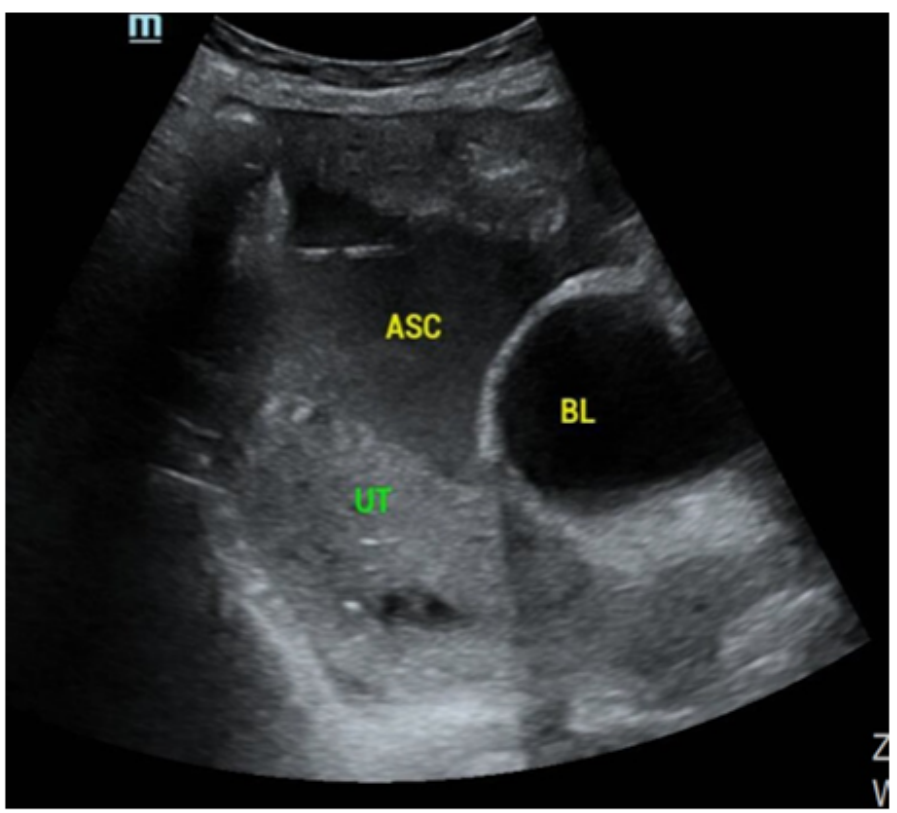

d

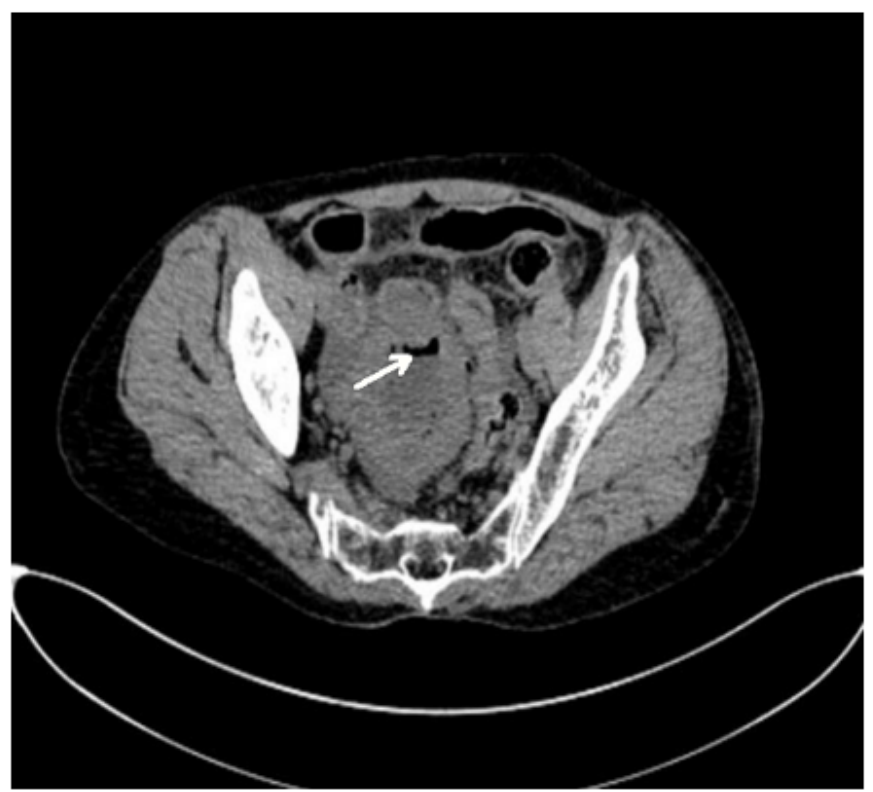

\section{Figure 1}

(a) Vertical abdominal radiograph showing free air under the diaphragm (white arrow). (b) Ultrasound examination showing uterine effusion, pelvic effusion, and ascites. (c) Abdominal CT showing free gas in the abdominal cavity (white arrow). (d) Abdominal CT showing fluid and gas in the uterine cavity (white arrow). 
$\mathbf{a}$

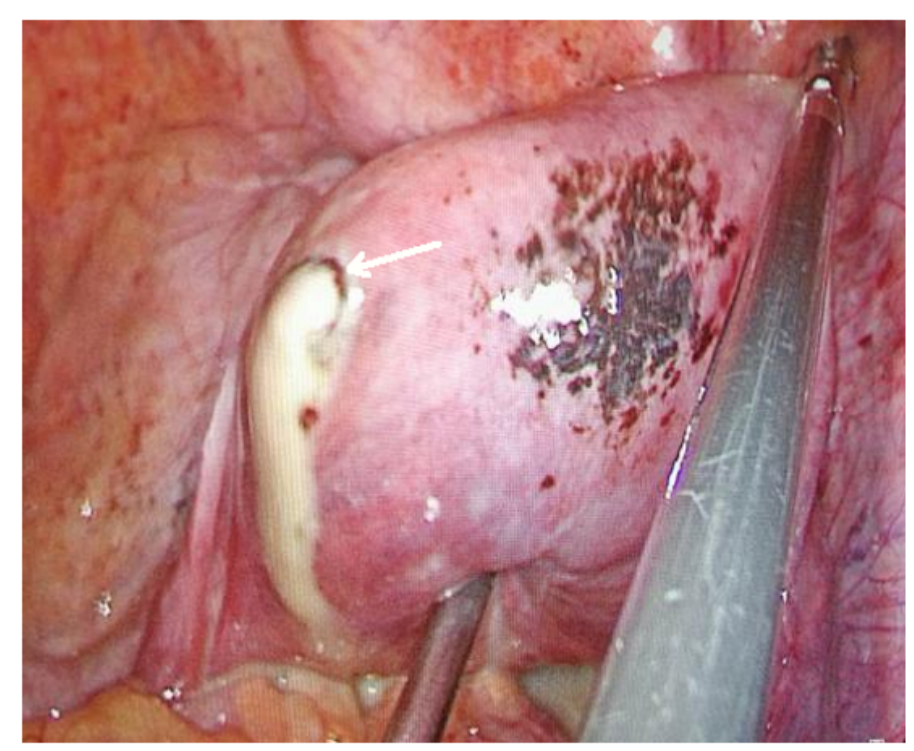

b

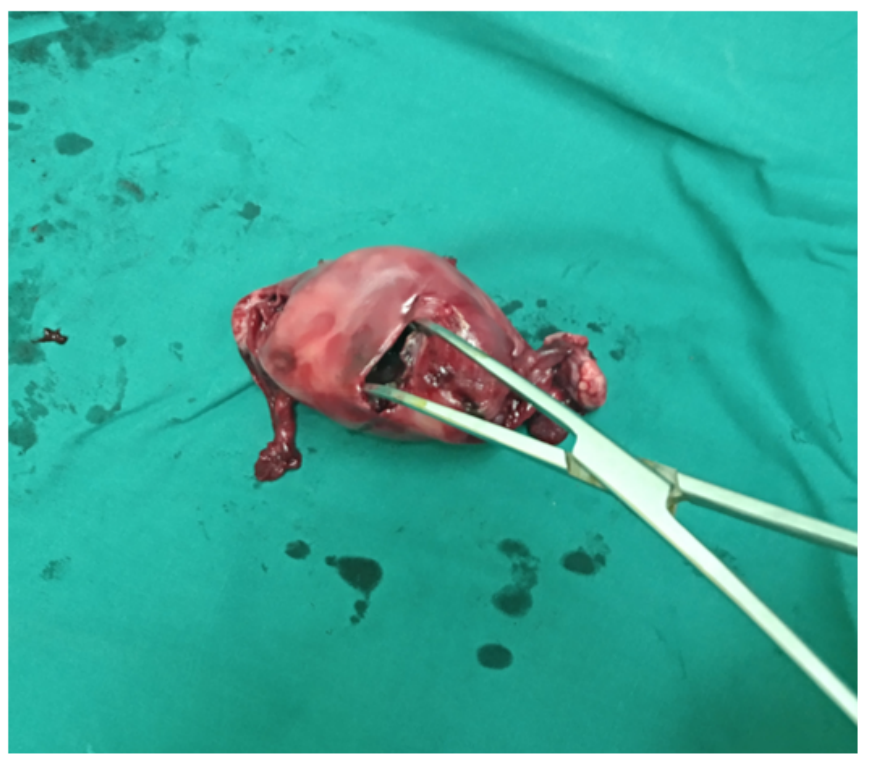

\section{c}

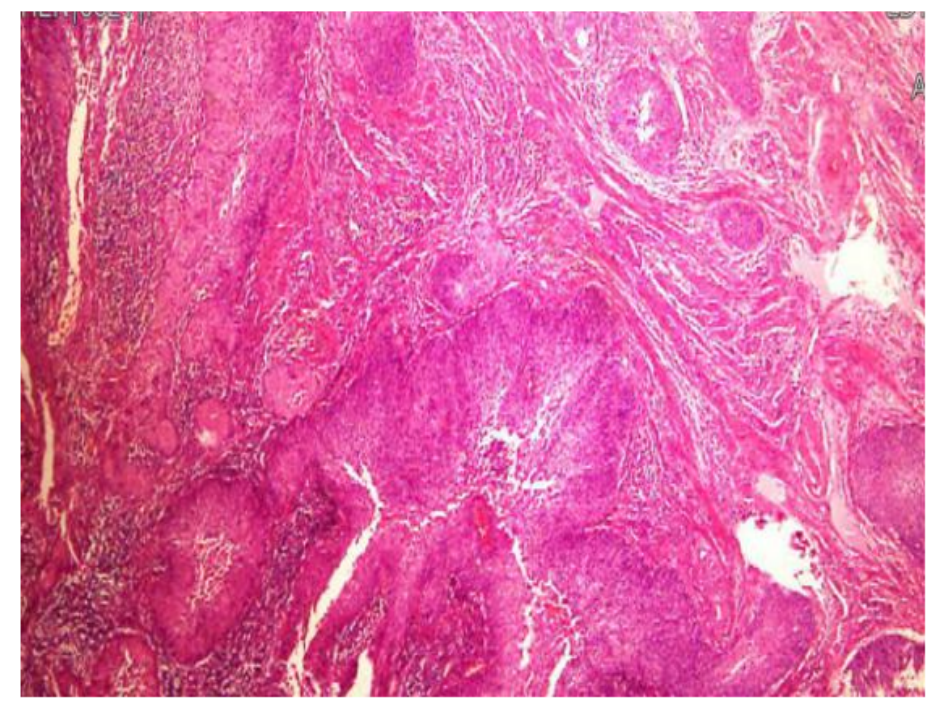

\section{Figure 2}

(a) A rupture can be seen in the left fundus of the uterus, with yellow-white pus continuously flowing out (white arrow). (b) Gross specimen. (c) Postoperative pathology. 